\title{
Deformation and Fracture Behaviours of Cementite
}

\author{
By Akihisa Inoue*, Tsugio Ogura* and Tsuyoshi Masumoto*
}

In order to clarify the deformation and fracture behaviours of cementite, observations by an optical microscope and a scanning electron microscope were made on the slip lines and fracture surfaces which took place around Vickers hardness indenters pressed on the surface of large cementite grain in white pig iron.

Although slip lines in cementite were generally straight, wavy slip lines and cross slip lines were occasionally observed under specified conditions of temperature and crystal orientation.

Large dependences on deformation temperature and crystal orientation were recognized for the slipping behaviour and hardness of cementite crystal. The slipping region increased and the hardness decreased with increase in deformation temperature. These temperature effects were remarkably large for cementites having the surface orientation around the center or near the [001 $]_{\theta^{-}}$ $[111]_{\theta}$ boundary of a stereographic triangle. The gliding of cementite having these orientations was very easy in comparison with those of the cementites having the orientation around $[001]_{\theta}$ or near the $[001]_{\theta}-[110]_{\theta}$ boundary of the stereographic triangle.

It was further made clear that the operation of the slip system of cementite crystal was closely related with the Schmid factor in spite of a strong anisotropy in the crystalline structure.

The fracture of cementite at room temperature was of a cleavage type, judging from the topographic features of fracture surfaces. The indeces of these fracture surfaces were determined to be $\{110\}_{\theta},\{100\}_{\theta}$ and $\{210\}_{\theta}$.

\section{(Received December 22, 1975)}

\section{Introduction}

Cementite is one of the most important constituents in steels. It is therefore very important to make clear the mechanical behaviour of cementite in understanding the mechanism of deformation and fracture of steels. From this viewpoint, in the previous paper ${ }^{(1)}$ the present authors examined the deformation and fracture of cementite in cold-rolled carbon steels by using a transmission electron microscope, and showed that cementite in steels was deformed in the same manner as dislocation gliding even at room temperature. In this paper, some experimental results of the slip system and fracture process of cementite in steels were also presented.

It has been known empirically that cementite in steels is highly deformable at elevated temperatures $^{(2)}$. However, the deformability of cementite itself has hardly been known because a large difficulty exists in preparing a cementite crystal which has enough dimensions to test its mechanical properties and to examine its

* The Research Institute for Iron, Steel and Other Metals, Tohoku University, Sendai 980, Japan. deformation behaviour. In the present study, trials were made at various temperatures to measure Vickers hardness and to observe slip markings and cracks around the Vickers indentations for cementite crystals with various orientations. One of the purposes of the present experiment is to clarify the effects of temperature and crystal orientation on the slipping behaviour and hardness of cementite which is possessed of a strong anisotropy ${ }^{(3)}$ in the crystalline structure. The other is to clarify the fracture plane and the topographic feature of the fractured surface of cementite.

\section{Materials and Experimental Procedures}

The material used in the present experiment is a white pig iron having the chemical composition of $\mathrm{Fe}-4.81 \% \mathrm{C}-5.80 \% \mathrm{Cr}^{\dagger}$. The pig iron was prepared by induction melting from electrolytic iron, electrolytic chromium and pure graphite in an argon atmosphere. The ingot of the pig iron was remelted and solidified unidirectionally at the speed of $3 \mathrm{~cm} / \mathrm{hr}$ in an

$\dagger$ Chromium was added to suppress the graphitization of cementite during the unidirectional solidification at very slow cooling rate.

1976 Vol. 17 
argon atmosphere to get cementite crystals larger than about $1 \mathrm{~mm}$ in diameter. The unidirectionally solidified ingot was cut into columns of $9 \mathrm{~mm}$ in diameter and $5 \mathrm{~mm}$ in height by spark cutting. These columns were then electropolished in an electrolyte containing $100 \mathrm{~g}$ of chromic acid and $200 \mathrm{~m} \ell$ of phosphoric acid to remove the strain produced by the cutting procedure. Vickers indenter was pressed on the surface of cementite crystal under a load of $500 \mathrm{~g}$ or $1 \mathrm{~kg}$ at various temperatures ranging from room temperature to $700^{\circ} \mathrm{C}$ in an argon atmosphere by using a Vickers hardness tester equipped with the hot stage. Observations were carried out on slip lines formed around the indentation by using a differential interference optical microscope (Union MED-M type). The surface orientation of cementite crystal was determined by means of the micro-Laue method.

The cementite crystal was fractured by a Vickers indenter under a load of $5 \mathrm{~kg}$ at room temperature, and the fracture feature was examined by a JSM-U3 type scanning electron microscope having the tilting device of $\pm 45^{\circ}$.

\section{Experimental Results and Discussions}

\section{Temperature dependence of hardness}

The temperature dependence of Vickers microhardness of cementite in the unidirectionally solidified $\mathrm{Fe}-4.81 \% \mathrm{C}-5.80 \% \mathrm{Cr}$ alloy is shown in Fig. $1^{\dagger}$, where the hardness in logarithmic scale is plotted against the reciprocal of absolute temperature. A linear relationships exist in the temperature ranges both below $220^{\circ} \mathrm{C}$ and above $420^{\circ} \mathrm{C}$. The hardness starts to decrease gradually at about $220^{\circ} \mathrm{C}$ and exhibits a steep decrease at temperatures above $420^{\circ} \mathrm{C}$ indicating that the plastic deformation of cementite is very easy at the temperature range. Such a temperature dependence of cementite hardness is in good agreement with that found by Yakushiji et al. ${ }^{(4)}$

\section{Slip planes at room temperature}

Optical micrographs of slip lines formed

The hardness shown in Fig. 1 are the average values obtained on several cementites with various surface orientations.

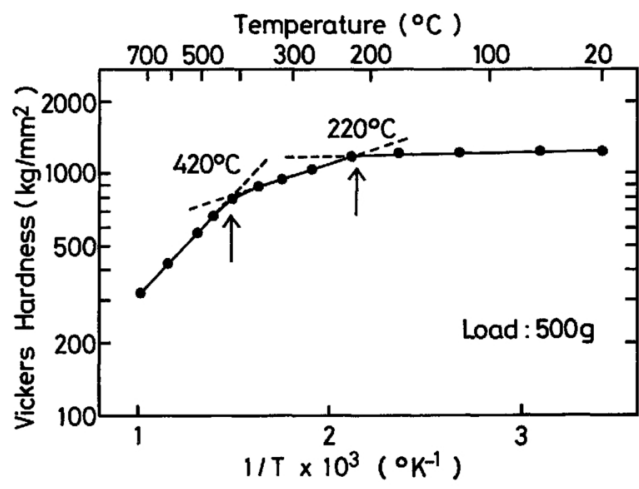

Fig. 1 Effect of temperature on the hardness of cementites in $\mathrm{Fe}-4.81 \% \mathrm{C}-5.80 \% \mathrm{Cr}$ cast iron.

around indentations on the surfaces of two cementite crystals are shown in Photo. 1(a) and (b). From Photo. 1 it is shown that a cementite is deformed by slipping, i.e. via dislocation gliding, even at room temperature. The following features of slip markings are further apparent: (1) More than two kinds of slip systems are operative; (2) Cross-slipping takes place as indicated by an arrow; (3) Wavy slip lines similar to those of $\alpha$-iron ${ }^{(5)}$ are observed.

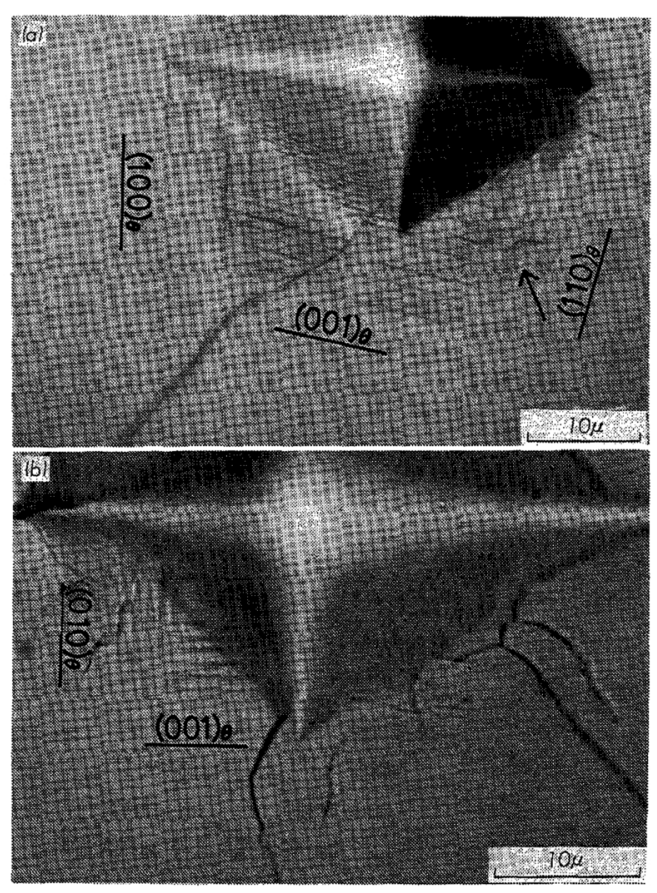

Photo. 1 Optical micrographs showing the slip lines formed around indentations on the surfaces of three cementite crystals at room temperature. 
In Fig. 2, their surface orientations are shown in the standard $(001)_{\theta}$ stereographic projection by marks a and $b$, respectively. The trace analyses of the slip lines indicate that operative slip planes of the cementite at room temperature are $(100)_{\theta},(010)_{\theta},(001)_{\theta}$ and $(110)_{\theta}$. Among these planes, the first three planes had already been confirmed with cementite in steels subjected to cold-rolling ${ }^{(1)}$. The operation of $(110)_{\theta}$ may be due to the high level of elastic strain concentration around a Vickers indentation ${ }^{(6)}$.

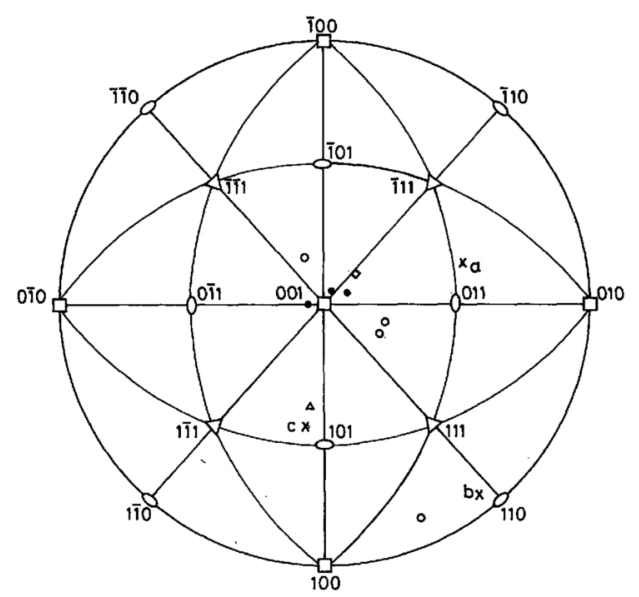

Fig. 2 Standard $(001)_{\theta}$ stereographic projection showing the surface orientations of cementite crystals picked up for the examination of effects of deformation temperature and crystal orientation.

\section{Dependences of slipping behaviour and hardness on temperature and crystal orientation}

The surface orientations of cementite crystals used for the present observation are shown in Fig. 2. They are roughly classified into four kinds; the first is around [001] $]_{\theta}$ (solid circles), the second around the center of stereographic triangle (open circles), the third near the $[001]_{\theta}-[101]_{\theta}$ boundary of the stereographic triangle (triangles), and the fourth along the $[001]_{\theta}-[\overline{1} 11]_{\theta}$ boundary (squares).

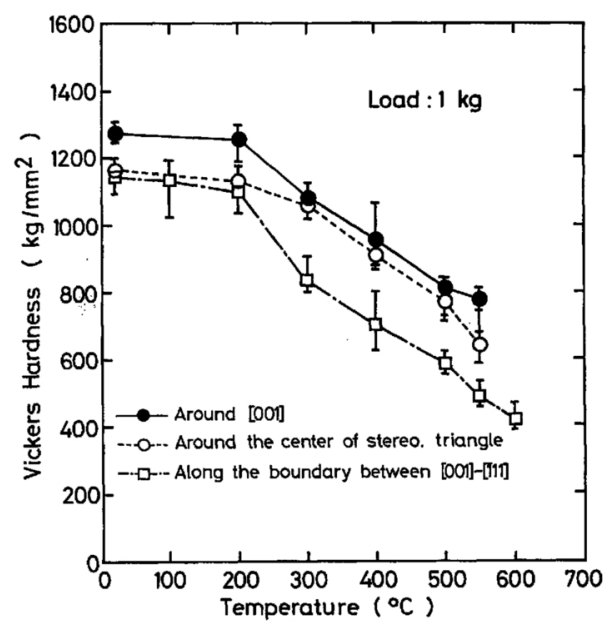

Fig. 3 Temperature dependence of the hardness of cementite crystals with various surface orientations.

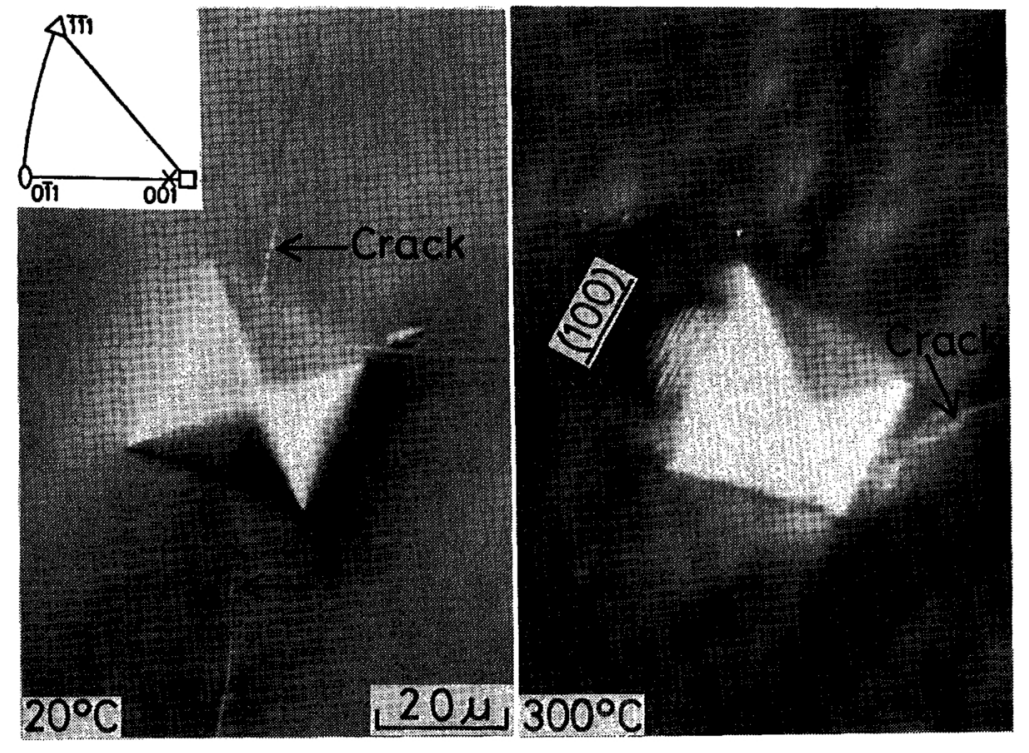

Photo. 2 Optical micrographs showing the slip lines formed on the cementite surface having the orientation around $[001]_{\theta}$. 

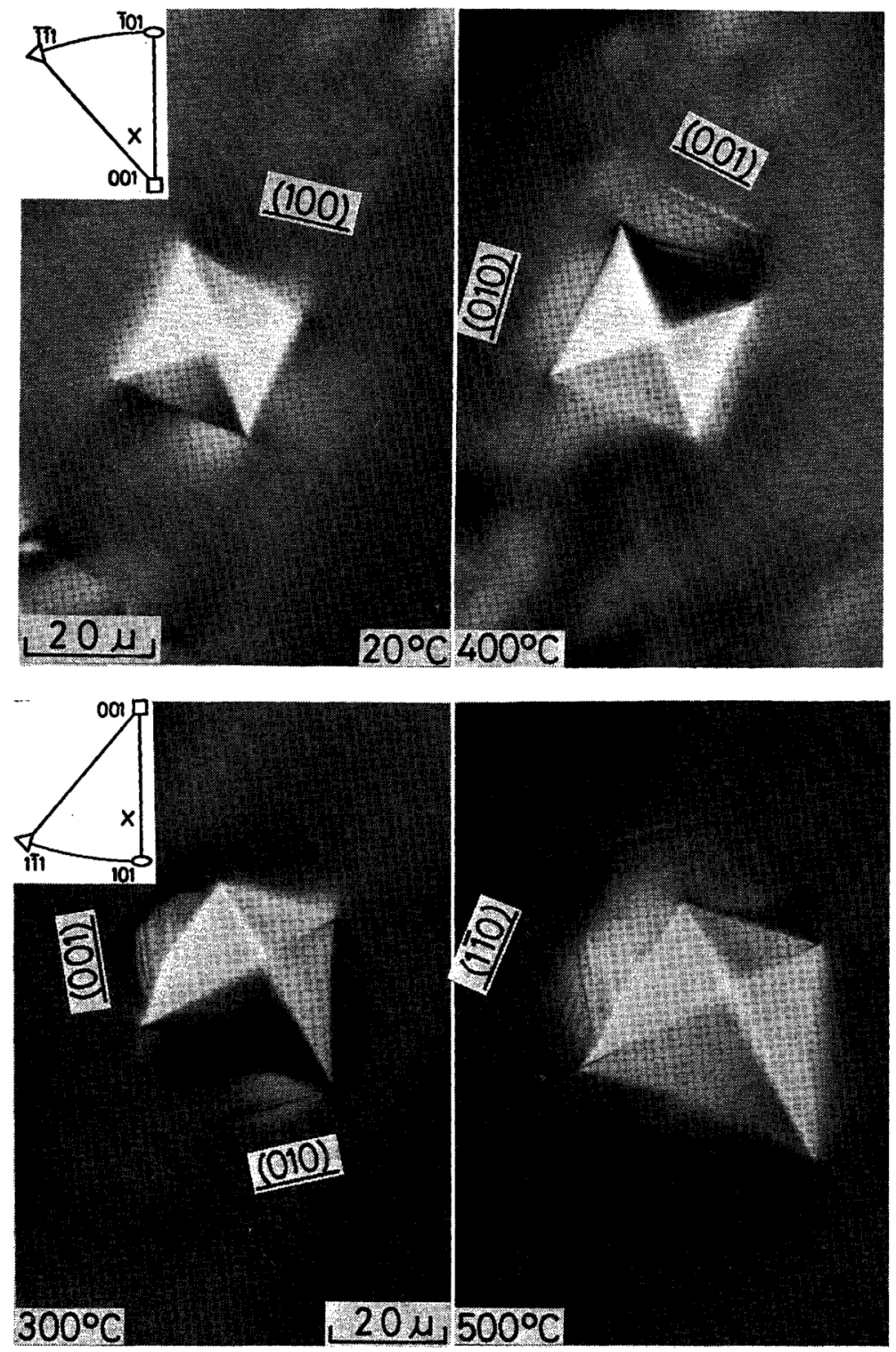

Photo. 3 Optical micrographs showing the slip lines formed on the cementite surface having the orientation around the center of a stereographic triangle.
Photo. 4 Optical micrographs showing the slip lines formed on the cementite surface having the orientation along the boundary between $[001]_{\theta^{-}}$ $[101]_{\theta}$.
The temperature dependences of hardness for cementite crystals with various surface orientations are shown in Fig. 3. The hardness of cementite measured on the surface which has the orientation of around $[001]_{\theta}$ is highest at all temperatures examined, while the one having the orientation along the boundary between $[001]_{\theta}-[111]_{\theta}$ is relatively soft and shows a large temperature dependence. Thus, the plasticity of cementite crystal is strongly affected by crystal orientation and temperature.

Optical micrographs of slip lines at various temperatures are shown in Photo. 2 for the cementite having a surface orientation around $[001]_{\theta}$. At room temperature, no distinct slip line is observed. At $300^{\circ} \mathrm{C}$, distinct slip lines are often observed along the traces of $(100)_{\theta}$ and $(010)_{\theta}$ and accompanied few cracks. As the temperature increases up to $500^{\circ} \mathrm{C}$, the formation of slip lines becomes easier and the maximum length ${ }^{\dagger}$ of the slip line measured from the edge of indentation increases. It is thus clear that with a $[001]_{\theta}$ orientation, a

$\dagger$ The method for measuring the maximum length of slip lines from Vickers indentation is illustrated in Fig. 4. 
very small amount of glide is followed by cracking at room temperature, whereas the gliding becomes comparatively easier at elevated temperatures.

The slipping features of the cementite crystal having a surface orientation around the center of a stereographic triangle are represented in Photo. 3. The cementite with this surface orientation exhibits in general a distinct deformability even at temperatures lower than $300^{\circ} \mathrm{C}$. This inclination is especially strong at temperatures above $400^{\circ} \mathrm{C}$ as is evident in this photograph. These observations indicate that the extent of gliding and its temperature dependence in the crystal with this orientation is larger than those having the orientation around $[001]_{\theta}$.

Photograph 4 shows the slipping behaviours of the cementite having a surface orientation along the $[001]_{\theta}-[101]_{\theta}$ boundary. In this cementite, although a few cracks occur, distinct slip lines along the traces of $(001)_{\theta}$ and $(010)_{\theta}$ planes are observed at temperatures below $300^{\circ} \mathrm{C}$. At $500^{\circ} \mathrm{C}$, in addition to the $(001)_{\theta}$ and $(010)_{\theta}$ planes, the operation of $(110)_{\theta}$ plane is observed. Photograph 5 shows the slipping behaviour of the cementite having a surface orientation near the $[001]_{\theta}-[\overline{1} 11]_{\theta}$

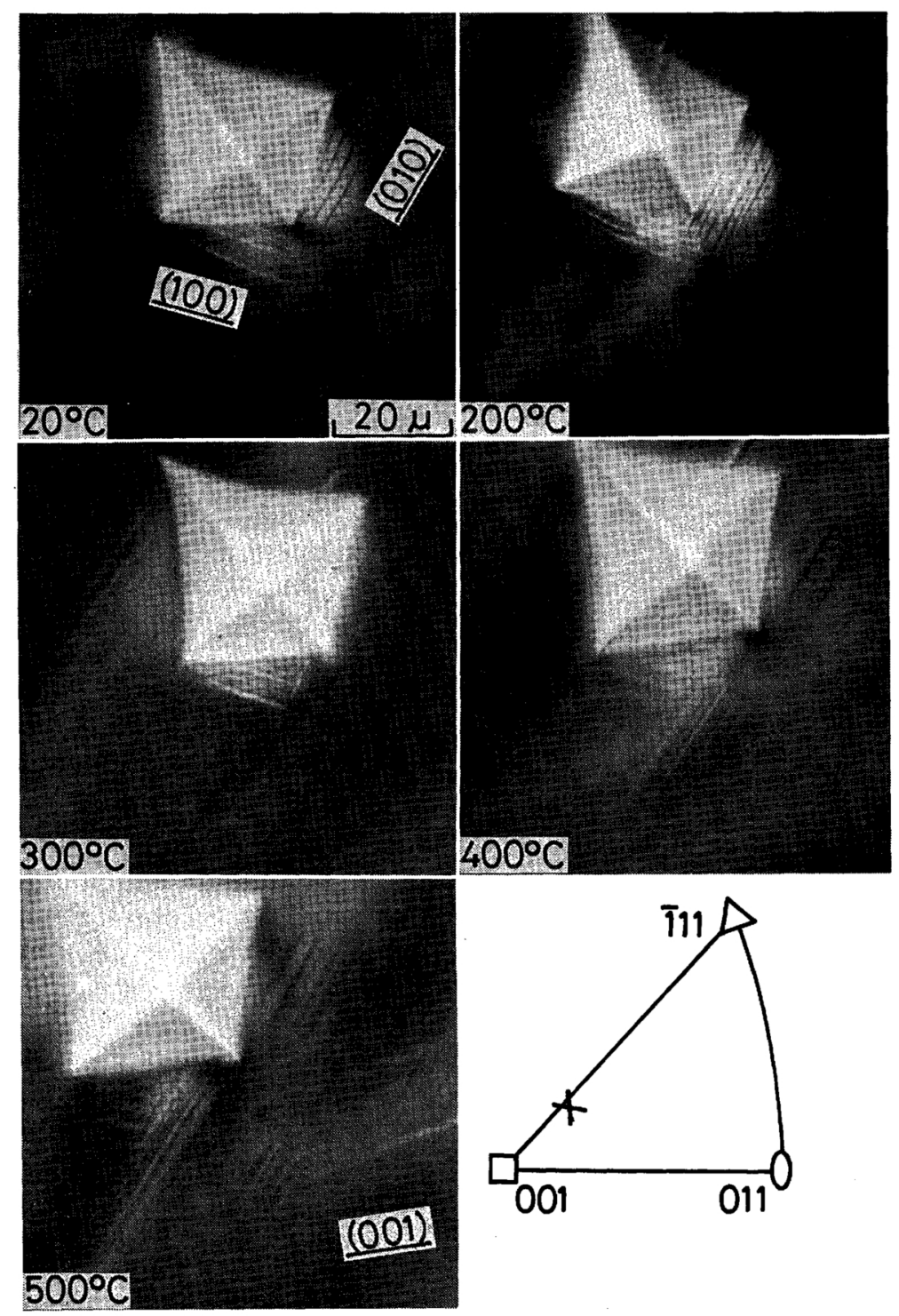

Photo. 5 Optical micrographs showing the slip lines formed on the cementite surface having the orientation near the boundary between $[001]_{\theta}-[\overline{1} 11]_{\theta}$. 


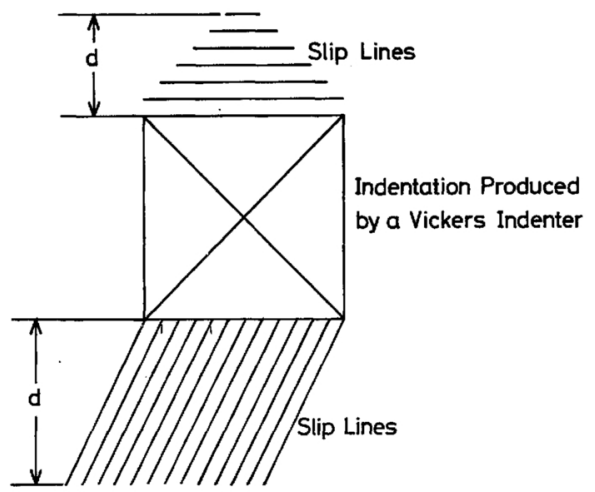

Fig. 4 Schematic diagram illustrating the maximum length of slip lines, $d$, measured from the edge of Vickers indentation.

boundary. As seen in the photographs, both $(100)_{\theta}$ and $(010)_{\theta}$ operate at room temperature and $200^{\circ} \mathrm{C}$. At $300^{\circ} \mathrm{C}$, the length of the individual slip line and the slipping region considerably increase. At $400^{\circ} \mathrm{C}$, the $(001)_{\theta}$ plane operates together with the $(100)_{\theta}$ and $(010)_{\theta}$ planes. As the temperature rises up to $550^{\circ} \mathrm{C}$, the slipping region becomes larger. The slipping behaviour and its temperature dependence observed on this surface are roughly similar to those having the orientation

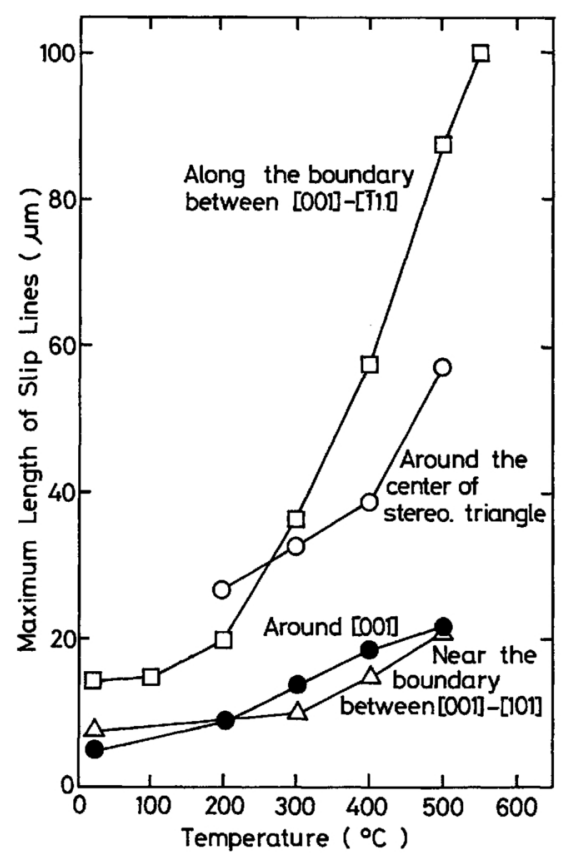

Fig. 5 Variation of maximum length of slip lines measured from the edge of Vickers indentation with deformation temperature for cementite crystals having various surface orientations. around the center of the stereographic triangle.

The maximum length of slip lines measured from the edge of Vickers indentation is shown in Fig. 5 as functions of the temperature and the crystal orientation. As seen in the figure, the gliding of cementite having the orientation around the center of the stereographic triangle (i.e. $[218]_{\theta}$ or $[116]_{\theta}$ ) becomes easy above $200^{\circ} \mathrm{C}$. However, the gliding of cementites having the orientations around $[001]_{\theta}$ and near the $[001]_{\theta^{-}}$ $[101]_{\theta}$ boundary is difficult even at temperatures higher than about $200^{\circ} \mathrm{C}$.

As described already, the slipping behaviour and hardness of cementite crystal are affected strongly by the deformation temperature and crystal orientation, but the gliding in cementite generally becomes easy above about $200^{\circ} \mathrm{C}$ except a confined surface orientation. This tendency agrees qualitatively with the temperature dependence of the hardness of cementite as shown in Figs. 1 and 3.

\section{Relationship between operative slip plane and Schmid factor}

As mentioned above, the slipping behaviours of cementite crystal is remarkably affected by deformation temperature and crystal orientation. The origin of the effect of crystal orientation is assumed to be due to either an anisotropy in crystal structure or a difference in the Schmid factor of operative slip systems.

The importance of the Schmid factor was examined by a following experiment. When a Vickers indenter is pressed on a specimen surface, a thin layer of the surface receives a compressive force, which is parallel to the specimen surface and normal to the edge of the indentation ${ }^{(7)(8)}$. In the present experiment, therefore, a Vickers indenter was used in order to change the Schmid factor of operative slip systems. The indenter was first pressed to a cementite with $1 \mathrm{~kg}$, then after rotating the specimen by $45^{\circ}$ around the normal to the surface the same indenter was again pressed with the same force. The surface orientation of the specimen used was $[421]_{\theta}$, and the directions $(A, B)$ of the surface forces by the first indentation and those $(C, D)$ by the second indentation are shown in a stereographic projection of Fig. 6.

The result obtained at $500^{\circ} \mathrm{C}$ is shown in 
Photo. 6. In the regions which receive the forces of the B, C and D directions, a large number of slip lines are seen in several kinds of slip systems. However, in the region which receives the force of the A direction, no operation of the slip system is recognized. This inclination was also recognized at room temperature and at $400^{\circ} \mathrm{C}$. Schmid factors of several slip systems were calculated for each case of directions A, B, C and D. The results are shown in Table 1 , where the slip planes

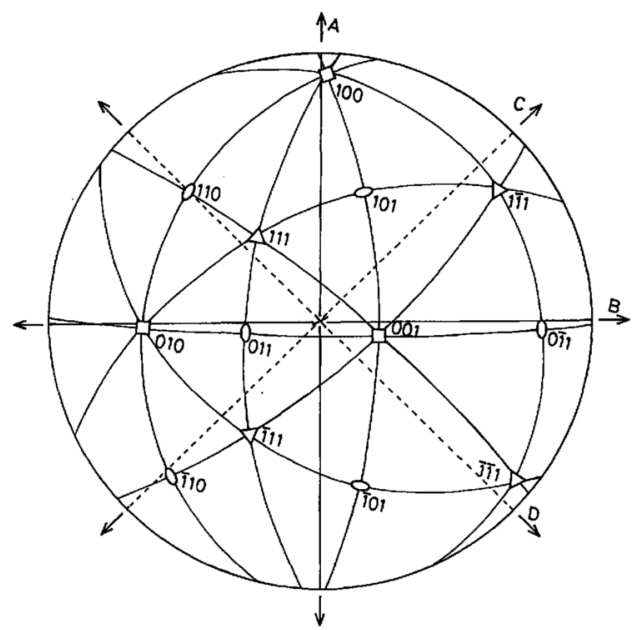

Fig. 6 Stereographic projection showing the directions of surface forces during the first indentation $(A, B)$ and those during the second indentation $(C, D)$ for the cementite having a surface orientation around $[421]_{\theta}$. observed in the present investigation are all listed. The slip directions marked asterisks in the table have been determined by the dark field analysis in a transmission electron microscopy ${ }^{(1)(9)(10)}$. The others are the most closepacked direction on each slip plane. As is evident from the table, all slip systems with some exceptions have large Schmid factors when a force is exerted along the B, C or D direction. On the other hand, when a force is exerted along the A direction, the Schmid factor for each slip system is generally very small. These calculated results are in good agreement with the aforementioned slipping behaviour shown in Photo. 6 ; that is, the slip-

Table 1 Schmid factors of several slip systems for each case of the directions A, B, C and D.

\begin{tabular}{lcccc}
\hline \hline & $\mathrm{A}$ & \multicolumn{1}{c}{$\mathrm{B}$} & \multicolumn{1}{c}{$\mathrm{C}$} & \multicolumn{1}{c}{$\mathrm{D}$} \\
\hline Slip System & $\begin{array}{c}\cos \mathbf{P} \cdot \\
\cos \lambda\end{array}$ & $\begin{array}{l}\cos \mathbf{P} \cdot \\
\cos \lambda\end{array}$ & $\begin{array}{l}\cos \mathbf{P} \cdot \\
\cos \lambda\end{array}$ & $\begin{array}{l}\cos \mathbf{P} \\
\cos \lambda\end{array}$ \\
\hline$(100)[010]^{*}$ & 0.035 & 0.032 & 0.481 & 0.429 \\
$(001)[010]^{*}$ & 0.004 & 0.372 & 0.139 & 0.244 \\
$(010)[001]^{*}$ & 0.004 & 0.372 & 0.139 & 0.244 \\
$(010)[100]$ & 0.035 & 0.032 & 0.481 & 0.429 \\
$(110)[001]$ & 0.068 & 0.267 & 0 & 0.334 \\
$(110)[001]$ & 0.074 & 0.278 & 0.203 & 0.006 \\
$(101)[010]$ & 0.026 & 0.237 & 0.481 & 0.482 \\
$(101)[010]$ & 0.031 & 0.175 & 0.314 & 0.225 \\
$(011)[100]$ & 0.087 & 0.034 & 0.293 & 0.199 \\
$(01 \overline{1})[100]$ & 0.035 & 0.018 & 0.481 & 0.490 \\
\hline \hline
\end{tabular}
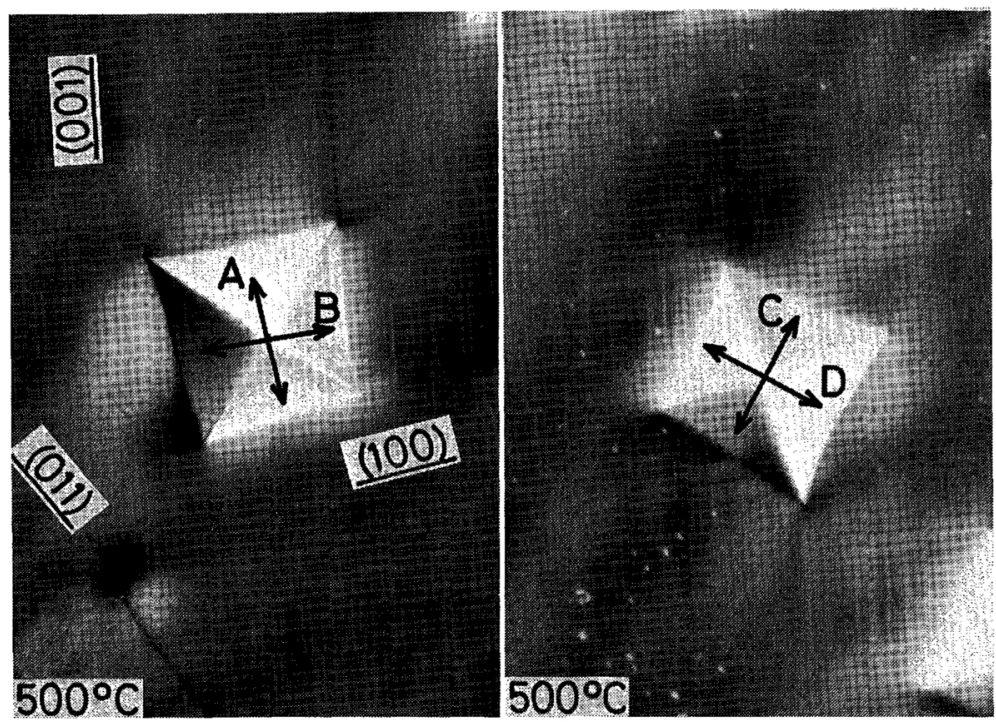

Photo. 6 Slip lines formed around two indentations which are twisted by $45^{\circ}$ from each another on the same specimen surface to show the effect of difference in the direction of surface forces around the indentations. 
ping activity is large in the region where the slip system has a large Schmid factor. It is therefore suggested that, even in a cementite crystal having a strong anisotropy ${ }^{(3)}$, the activity of slipping and Schmid factor are closely related with each another.

\section{Cleavage fracture}

Scanning electron micrographs of a fracture surface of a specimen having the surface orientation around $[001]_{\theta}$ are shown in Photo. 7(a) and (b). In Photo. 7(a), a fracture facet (arrow) with a smooth appearance is visible along the trace of $(\overline{101})_{\theta}$ plane. Photograph 7 (b) was taken from the same area as (a) after tilting the specimen by $45^{\circ}$. Both the axis and the direction of the tilting are illustrated in Fig. 7. The area of the fracture surface becomes smaller with the tilting, and after tilting by $45^{\circ}$ the shape of the fracture facets becomes linear as seen in Photo. 7(b). Based on the above observation, the fracture plane was indexed through the procedure indicated in the stereographic projection of cementite in Fig. 7. $\mathrm{C}$ in the figure shows the original orientation of the specimens surface. The directions $A-B$ and $a-b$ in the figure indicate the trace of the fracture facet and the rotation axis of the specimen, respectively. The viewing direction originally matched with the surface orientation of the specimen is shifted to the position $\mathrm{D}$ after tilting by $45^{\circ}$. The projection point of the normal of the fracture facet should be in the direction normal to the direction $\mathrm{A}-\mathrm{B}$ and at $90^{\circ}$ from the position $\mathrm{D}$. The projection point which satisfied these condi- tions is just $(\overline{101})_{\theta}$. The fracture plane seen in Photo. 7(a) is thus determined to be $(101)_{\theta}$.

The appearance of the fracture surface of cementite with an other surface orientation is shown in Photo. 8(a) and (b). In Photo. 8(a), many linear steps are seen on the fracture surface along the $(0 \overline{1} 1)_{\theta}$ trace. Photograph 8(b) was taken from the same area as (a) after tilting by $40^{\circ}$. It is noticeable that the steps rotates clock-wise with the tilting. Figure 8 shows the stereographic projection in which the center is the projection point of the viewing direction. The tilting axis and its direction are also indicated. The marks $(\leftrightarrow)$ indicate the

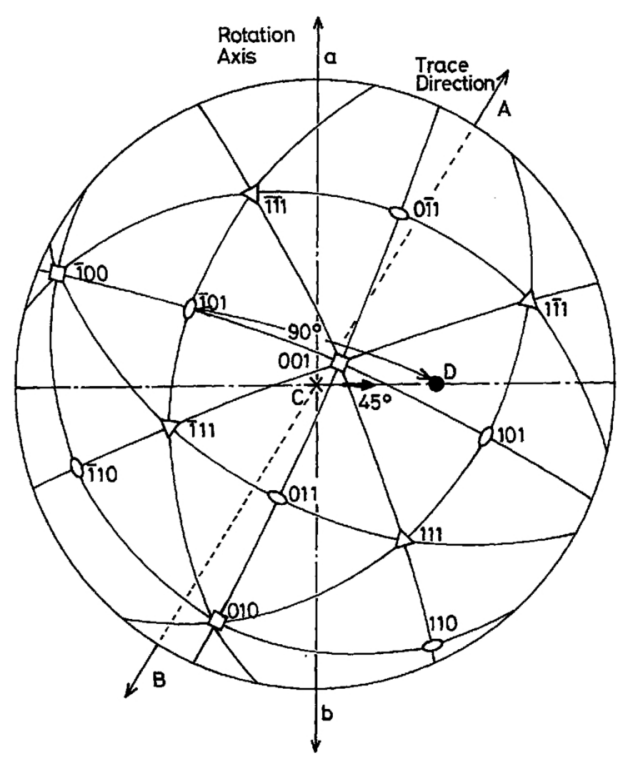

Fig. 7 Stereographic projection of cementite showing the procedure for the indexing of fracture planes shown in Photo. 7.
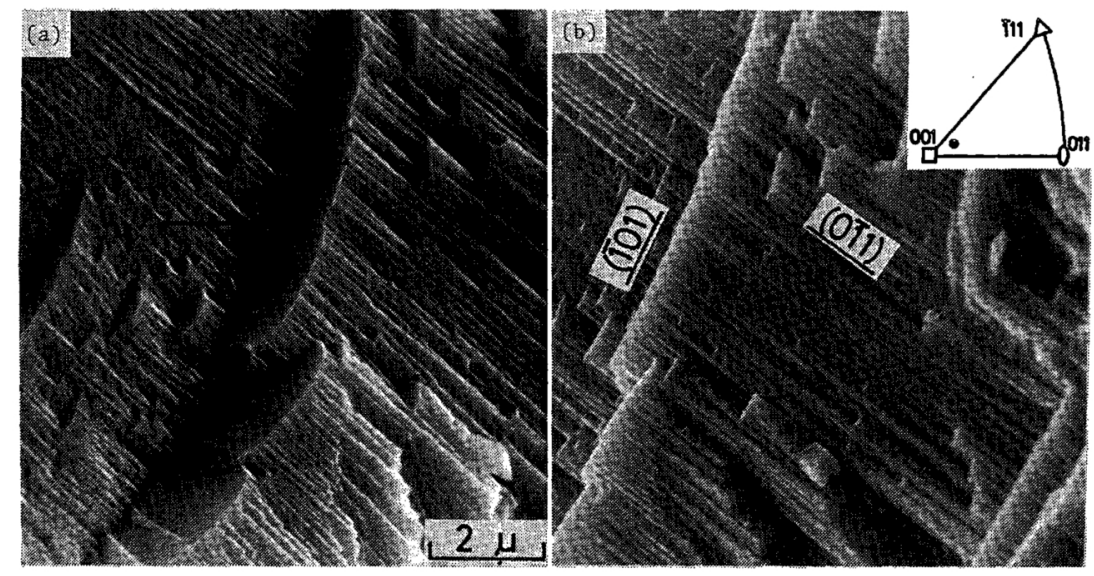

Photo. 7 Scanning electron micrographs showing the fracture surface of cementite crystal, and the stereographic triangle showing the surface orientation. 


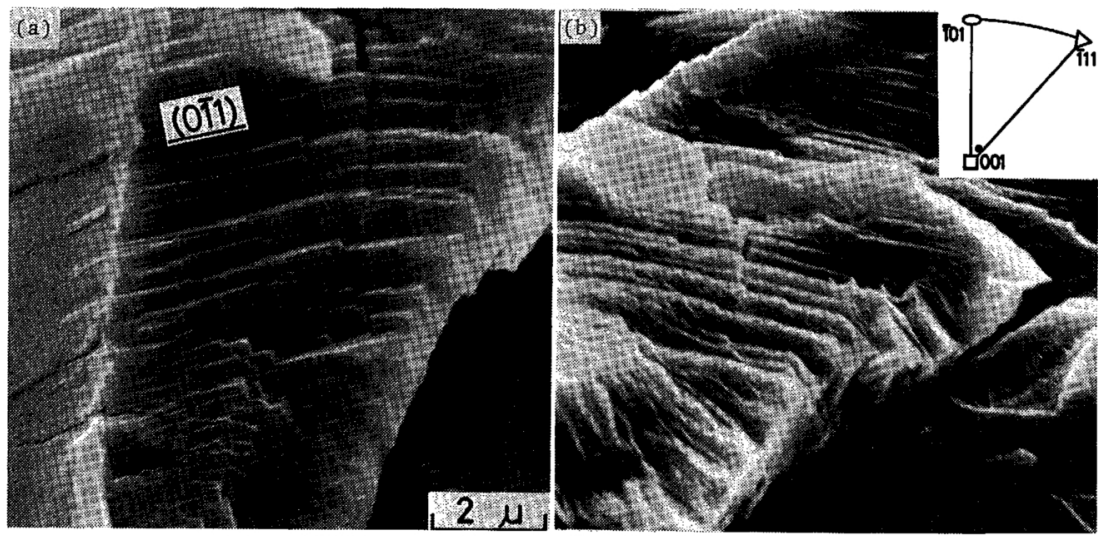

Photo. 8 Scanning electron micrographs showing the cleavage steps of cementite crystal, and the stereographic triangle showing the surface orientation.

elongated directions of the surface steps. The shift of the $(0 \overline{1} 1)_{\theta}$ projection points after tilting by $20^{\circ}$ and $40^{\circ}$ are also indicated, respectively. Changes in the elongated direction of the surface steps are reasonably explained only by assuming that the surface step is $(0 \overline{1} 1)_{\theta}$. This suggests that their cleavage steps are formed by the cleavage fracture on the $(0 \overline{1} 1)_{\theta}$ plane.

Further examinations were made on many other fracture surfaces, and showed that fractures occur mostly along the $\{110\}_{\theta}$ plane. A few fractures along $\{100\}_{\theta}$ and $\{210\}_{\theta}$ are also found.

Another topographic features of fracture surface are shown in Photo. 9(a) and (b). As seen in these photographs, fine wavy markings or river markings are found on almost all fracture surfaces accompanying to the cleavage type fracture. The result suggests that some amount of mechanical energy due to plastic flow is dissipated near the fracture surfaces.

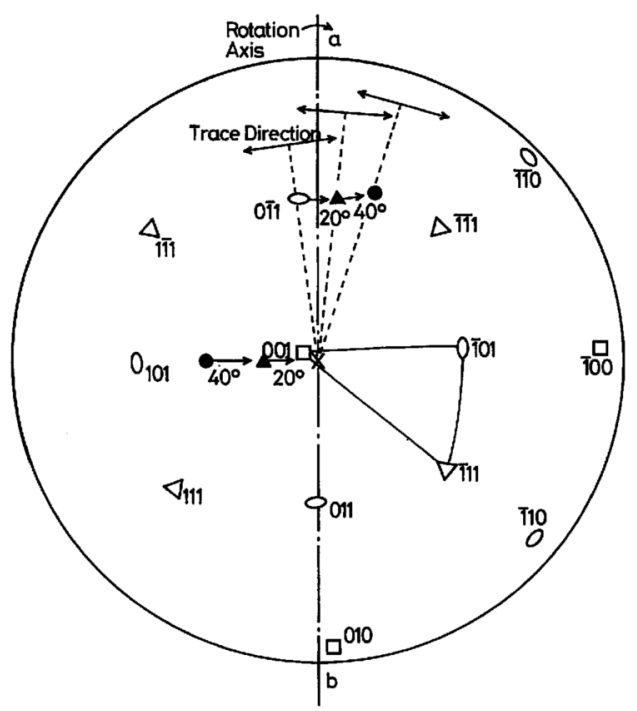

Fig. 8 Stereographic projection of cementite showing the procedure for the indexing of cleavage steps shown in Photo. 8.

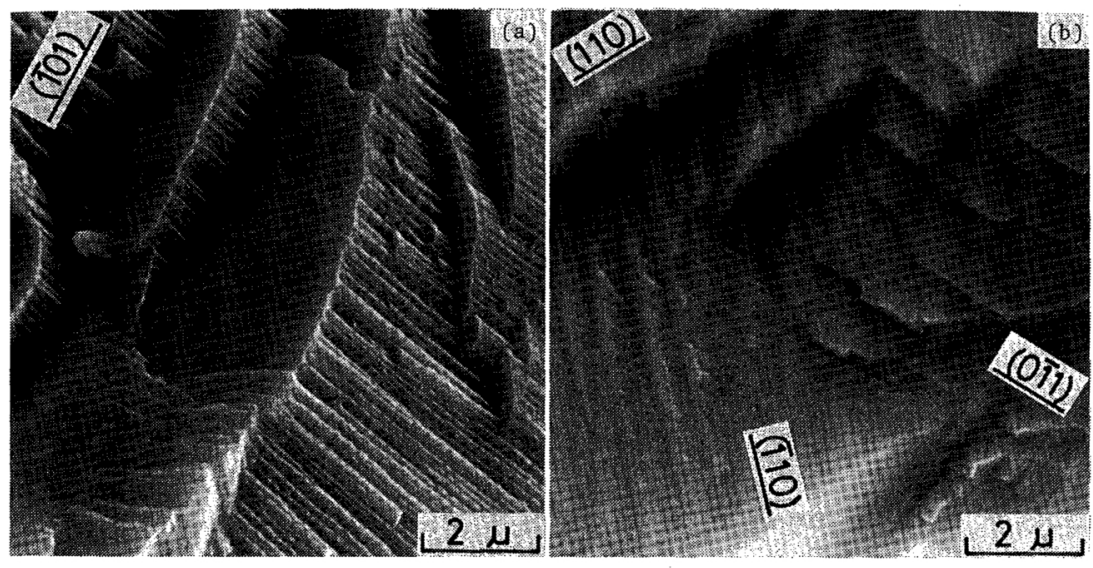

Photo. 9 Scanning electron micrographs showing the topography of fracture surfaces. 


\section{Summary}

Optical microscope and scanning electron microscope observations were made on the slip lines and fracture surfaces which appeared around Vickers indentations pressed on the surface of a large cementite crystal in white pig iron. The hardness of cementite was also measured. The results obtained are summarized as follows:

(1) The hardness of cementite decreased gradually at temperatures above $220^{\circ} \mathrm{C}$, and decreased steeply at temperatures higher than $420^{\circ} \mathrm{C}$.

(2) Distinct slip lines were observed around indentations on the surface of a cementite crystal. The slip lines generally appeared straight, but wavy slip lines similar to those of $\alpha$-iron were also observed.

(3) The hardness of cementite exhibited large dependences on temperature and crystal orientation. The cementite having the surface orientation along the $[001]_{\theta}-[\overline{1} 11]_{\theta}$ boundary was soft and had a large temperature dependence of hardness, while for cementite having the surface orientation around $[001]_{\theta}$ the hardness was highest and its temperature dependence was small.

(4) The slipping behaviour of cementite crystal exhibited large dependences on deformation temperature and crystal orientation. In the cementite having the surface orientation around $[001]_{\theta}$ no distinct slip lines were observed at room temperature due to stress relief by a preceding cracking. Above $300^{\circ} \mathrm{C}$, the cracking was suppressed and the glide on $\{100\}_{\theta}$ became operative. In the cementite having the surface orientation near the $[001]_{\theta^{-}}$ $[110]_{\theta}$, boundary the $\{100\}_{\theta}$ plane operated at room temperature. Above $400^{\circ} \mathrm{C},\{110\}_{\theta}$ plane was also active, and multiple gliding became possible.

The gliding of cementite having the orientation around the center or near the $[001]_{\theta}-[\overline{1} 11]_{\theta}$ boundary of the stereographic triangle was very easy in comparison with those of the orientation of the aforementioned kinds. In these cementites only $(100)_{\theta}$ plane was active at room temperature. At $200 \sim 300^{\circ} \mathrm{C}$, simultaneous gliding on $(100)_{\theta},(010)_{\theta}$ and $(001)_{\theta}$ were recognized. Above $400^{\circ} \mathrm{C}$, the $\{110\}_{\theta}$ plane became active to some extent.

(5) It was made clear that even though a cementite crystal has a strong anisotropy, the operation of its slip system was closely related with the Schmid factor.

(6) Topographic features of the cementite fracture surface were examined. The fracture occurred on $\{110\}_{\theta},\{100\}_{\theta}$ and $\{210\}_{\theta}$ planes. Fine wavy or river markings were found on almost all fracture surfaces. These results suggest that the fracture of cementite crystal was of the cleavage type.

\section{REFERENCES}

(1) A. Inoue, T. Ogura and T. Masumoto: J. Japan Inst. Metals, 37 (1973), 875; Trans. JIM, 17 (1976), 149.

(2) A. S. Keh: Acta Met., 11 (1963), 1101.

(3) N. J. Petch: J. Iron Steel Inst., 149 (1944), 143.

(4) M. Yakushiji, Y. Kondo, H. Matsumoto and T. Okamoto: J. Japan Inst. Metals, 39 (1975), 606.

(5) D. G. Brandon and J. Nutting: Acta Met., 7 (1959), 101.

(6) D. Tabor: The Hardness of Metals, Oxford at the Clarren Press, (1951), 101.

(7) M. Nakamura: The Hardness and Property of Metals, Japan Inst. Met., Symposium (December 7, 1973), 25.

(8) B. Lawn and R. Wilshaw: J. Mat. Sci., 10 (1975), 1049.

(9) A. Inoue, T. Ogura and T. Masumoto: Bull. Japan Inst. Metals, 13 (1974), 653.

(10) A. Inoue, T. Ogura and T. Masumoto: Scripta Met., to be published. 